\title{
RESEARCH
}

\section{Tropical ideals do not realise all Bergman fans}

\author{
Jan Draisma ${ }^{1,2^{*}}$ (i) and Felipe Rincón ${ }^{3}$
}

\author{
"Correspondence: \\ jan.draisma@math.unibe.ch \\ ${ }^{2}$ Eindhoven University of \\ Technology, Eindhoven, \\ Netherlands \\ Full list of author information is \\ available at the end of the article \\ JD was partially supported by the \\ Vici grant Stabilisation in Algebra \\ and Geometry from the \\ Netherlands Organisation for \\ Scientific Research (NWO) and \\ project grant 200021 191981 \\ from the Swiss National Science \\ Foundation (SNF). \\ FR was partially supported by the \\ Research Council of Norway \\ grant 239968/F20
}

\begin{abstract}
Every tropical ideal in the sense of Maclagan-Rincón has an associated tropical variety, a finite polyhedral complex equipped with positive integral weights on its maximal cells. This leads to the realisability question, ubiquitous in tropical geometry, of which weighted polyhedral complexes arise in this manner. Using work of Las Vergnas on the non-existence of tensor products of matroids, we prove that there is no tropical ideal whose variety is the Bergman fan of the direct sum of the Vámos matroid and the uniform matroid of rank two on three elements and in which all maximal cones have weight one.
\end{abstract}

\section{Introduction}

An ideal in a polynomial ring over a field with a non-Archimedean valuation gives rise to a tropical variety, either by taking all weight vectors whose initial ideals do not contain a monomial or, equivalently if the field and the value group are large enough [4, Theorem 4.2], by applying the coordinate-wise valuation to all points in the zero set of the ideal. In the middle of this construction sits a tropical ideal, obtained by applying the valuation to all polynomials in the ideal. This ideal is a purely tropical object, in that it does not know about the field or the valuation, and it contains more information than the tropical variety itself. For these reasons, tropical ideals, axiomatised in [6], were proposed as the correct algebraic structures on which to build a theory of tropical schemes. We review the relevant definitions below.

It was proved in [6] that tropical ideals, while not finitely generated as ideals-nor in any sense that we know of!- have a rational Hilbert series, satisfy the ascending chain condition, and define a tropical variety: a finite weighted polyhedral complex. Later in [7], it was shown that the top-dimensional parts of these varieties are always balanced polyhedral complexes. This leads to the following realisability question.

Question 1.1 Which pure-dimensional balanced polyhedral complexes are the variety of some tropical ideal?

If the tropical ideal is the tropicalisation of a prime classical ideal, then the tropical variety is pure-dimensional and balanced [8, Theorem 3.3.5]. The question of which bal-

(c) The Author(s) 2021. This article is licensed under a Creative Commons Attribution 4.0 International License, which permits use, sharing, adaptation, distribution and reproduction in any medium or format, as long as you give appropriate credit to the original author(s) and the source, provide a link to the Creative Commons licence, and indicate if changes were made. The images or other third party material in this article are included in the article's Creative Commons licence, unless indicated otherwise in a credit line to the material. If material is not included in the article's Creative Commons licence and your intended use is not permitted by statutory regulation or exceeds the permitted use, you will need to obtain permission directly from the copyright holder. To view a copy of this licence, visit http://creativecommons.org/licenses/by/4.0/. 
anced polyhedral complexes are realised by classical ideals has received much attention, especially in the case of curves (see e.g. $[2,3,13])$. But for general tropical ideals, very little is known about Question 1.1: for instance, no natural algebraic criterion that ensures that the variety is pure-dimensional is known. In fact, until recently we had no intuition as to whether tropical ideals are flexible enough that they can realise basically any balanced polyhedral complex, or rather more rigid, like algebraic varieties. In view of the following theorem, we now lean towards the latter intuition.

Theorem 5.2 Let $M$ and $N$ be loopless matroids of ranks $a$ and $b$ that do not have a quasi-product of rank $a \cdot b$. Then, there exists no tropical ideal whose tropical variety is the Bergman fan of the direct sum of $M$ and $N$, with all maximal cones having weight 1.

In particular, there exists no tropical ideal whose tropical variety is the Bergman fan of the direct sum of the Vámos matroid $V_{8}$ and the uniform matroid $U_{2,3}$ of rank two on three elements, with all maximal cones having weight 1.

In this theorem, a quasi-product of two loopless matroids is a matroid analogue of tensor products; see Sect. 4. The fact that the Vámos matroid $V_{8}$ and the uniform matroid $U_{2,3}$ have no quasi-product of rank 8 was proved by Las Vergnas in [5].

We believe that this theorem marks the beginning of an interesting research programme, which, in addition to the pureness and balancing questions mentioned above, asks which tropical ideals define matroids on the set of variables, and which matroids are, in this sense, tropically algebraic-See Problem 3.5 and Question 3.6.

\section{Definitions and basic results on tropical ideals}

Consider the tropical semifield $(\overline{\mathbb{R}}:=\mathbb{R} \cup\{\infty\}, \oplus, \odot)$ with $\oplus:=$ min and $\odot:=+$. Let $R$ be a sub-semifield of $\overline{\mathbb{R}}$. The example most relevant to us is the Boolean semifield $\mathbb{B}:=\{0, \infty\}$, which is not only a sub-semifield but also a quotient of $\overline{\mathbb{R}}$.

Definition 2.1 Let $N$ be a finite set. A set $L \subseteq R^{N}$ is a tropical linear space if it is an $R$-submodule (i.e. $(\infty, \ldots, \infty) \in L$ and $f, g \in L, c \in R \Rightarrow(c \odot f) \oplus g \in L)$ and if, moreover, $L$ satisfies the following elimination axiom: for $i \in N$ and $f, g \in L$ with $f_{i}=g_{i} \neq \infty$, there exists an $h \in L$ with $h_{i}=\infty$ and $h_{j} \geq f_{j} \oplus g_{j}$ for all $j \in N$, with equality whenever $f_{j} \neq g_{j}$. The $\overline{\mathbb{R}}$-submodule $L_{\overline{\mathbb{R}}}$ of $\overline{\mathbb{R}}^{N}$ generated by $L$ is a tropical linear space in $\overline{\mathbb{R}}^{N}$ and has the structure of a finite polyhedral complex; we denote its dimension as such by $\operatorname{dim} L$.

If $K$ is a field equipped with a non-Archimedean valuation onto $R$ and if $V \subseteq K^{N}$ is a linear subspace, then the image of $V$ under the coordinate-wise valuation is a tropical linear space in $R^{N}$, but not all tropical linear spaces arise in this manner. Tropical linear spaces are well-studied objects in tropical geometry and matroid theory: the definition above is equivalent to that of [12], except that we allow some coordinates to be $\infty$. A tropical linear space $L$ gives rise to a matroid $M(L)$ in which the independent sets are those subsets $A \subseteq N$ for which $L \cap\left(R^{A} \times\{\infty\}^{N \backslash A}\right)=\{\infty\}^{N}$, and $L$ is the set of vectors ( $R$-linear combinations of valuated circuits) of a valuated matroid on $M(L)$ [10]. With this setup, $\operatorname{dim} L=|N|-\operatorname{rk}(M(L))$. We will freely alternate between these different characterisations of tropical linear spaces.

Set $\mathbb{N}:=\{0,1,2, \ldots\}$, and let $n \in \mathbb{N}$. Denote by $R\left[x_{1}, \ldots, x_{n}\right]$ the semiring of polynomials in the variables $x_{1}, \ldots, x_{n}$ with coefficients in $R$. We write $\mathrm{Mon}_{\mathrm{d}}$ and Mon $\leq \mathrm{d}$ for the set of 
monomials in $x_{1}, \ldots, x_{n}$ of degree equal to $d$ and at most $d$, respectively, and we identify a polynomial in $R\left[x_{1}, \ldots, x_{n}\right]$ of degree at most $d$ with its coefficient vector in $R^{\text {Mon }} \leq \mathrm{d}$.

Definition 2.2 A subset $I \subseteq R\left[x_{1}, \ldots, x_{n}\right]$ is a tropical ideal if $x_{i} \odot I \subseteq I$ for all $i=1, \ldots, n$ and if for each $d \in \mathbb{N}$ the set $I_{\leq d}:=\{f \in I: \operatorname{deg}(f) \leq d\}$ is a tropical linear space in $R^{\mathrm{Mon}_{\leq \mathrm{d}}}$.

This definition is equivalent to [6, Definition 1.1]. Indeed, there, in addition to the requirement that $I_{\leq d}$ be a tropical linear space, it is required that $I$ is an ideal in the semiring $R\left[x_{1}, \ldots, x_{n}\right]$. This is equivalent to the statement that $I$ is closed under tropical multiplication by each $x_{i}$ and closed under tropical addition. However, as tropical linear spaces are already closed under tropical addition, this does not need to be included as an explicit axiom.

If $I$ is homogeneous, then the latter condition is equivalent to the condition that for each $d$ the set $I_{d}$ of homogeneous polynomials in $I$ of degree $d$ is a tropical linear space in $R^{\text {Mon }_{\mathrm{d}}}$. There is a natural notion of tropical ideals in the Laurent polynomial ring $R\left[x_{1}^{ \pm 1}, \ldots, x_{n}^{ \pm 1}\right]$ that we will also use, and if $I$ is a tropical ideal in $R\left[x_{1}, \ldots, x_{n}\right]$, then the set $I^{\prime}:=\left\{f / \mathbf{x}^{\mathbf{u}} \mid f \in I, \mathbf{u} \in \mathbb{N}^{n}\right\}$ is a tropical ideal in $R\left[x_{1}^{ \pm 1}, \ldots, x_{n}^{ \pm 1}\right]$.

Tropical ideals were introduced by Maclagan and Rincón in [6] as a framework for developing algebraic foundations for tropical geometry. Tropical ideals are much better behaved than general ideals of the polynomial semiring $R\left[x_{1}, \ldots, x_{n}\right]$, as we explain below.

Definition 2.3 For $\mathbf{w} \in \mathbb{R}^{n}$ and $f=\bigoplus_{\mathbf{u}} c_{\mathbf{u}} \odot \mathbf{x}^{\mathbf{u}} \in R\left[x_{1}, \ldots, x_{n}\right]$, define the initial part of $f$ relative to $\mathbf{w}$ as

$$
\operatorname{in}_{\mathbf{w}}(f):=\bigoplus_{\mathbf{u}: c_{\mathbf{u}}+\mathbf{u} \cdot \mathbf{w}=f(\mathbf{w})} \mathbf{x}^{\mathbf{u}} \in \mathbb{B}\left[x_{1}, \ldots, x_{n}\right]
$$

For a tropical ideal $I$, define its initial ideal relative to $\mathbf{w}$ as

$$
\operatorname{in}_{\mathbf{w}} I:=\left\langle\operatorname{in}_{\mathbf{w}} f \mid f \in I\right\rangle_{\mathbb{B}} .
$$

Note that in this paper we only consider weights $\mathbf{w}$ in $\mathbb{R}^{n}$, not in $\overline{\mathbb{R}}^{n}$ as in [6]. In other words, we do geometry only inside the tropical torus.

Definition 2.4 The Hilbert function of a tropical ideal $I \subseteq R\left[x_{1}, \ldots, x_{n}\right]$ is the map $H_{I}: \mathbb{N} \rightarrow \mathbb{N}$ given by $d \mapsto\left(\begin{array}{c}n+d \\ d\end{array}\right)-\operatorname{dim} I_{\leq d}$.

Note that, as usual in commutative algebra, the Hilbert function measures the codimension of $I_{\leq d}$ in its ambient space $R^{\text {Mon }_{\leq \mathrm{d}}}$. A homogeneous variant of this Hilbert function applies only to homogeneous ideals and measures the codimension of $I_{d}$ in $R^{\mathrm{Mon}_{\mathrm{d}}}$. The Hilbert function of a not necessarily homogeneous ideal $I$ in $R\left[x_{1}, \ldots, x_{n}\right]$ equals the homogeneous Hilbert function of its homogenisation in $R\left[x_{0}, \ldots, x_{n}\right]$.

The following is a special case of [6, Corollary 3.6].

Theorem 2.5 For a homogeneous tropical ideal $I \subseteq R\left[x_{1}, \ldots, x_{n}\right]$ and any $\mathbf{w} \in \mathbb{R}^{n}$, $\operatorname{in}_{\mathbf{w}} I \subseteq \mathbb{B}\left[x_{1}, \ldots, x_{n}\right]$ is a homogeneous tropical ideal, and $H_{\mathrm{in}_{\mathbf{w}} I}=H_{I}$.

Theorem 2.5 allows one to pass to monomial initial ideals and show that the Hilbert function $H_{I}(d)$ of a homogeneous tropical ideal $I$ becomes a polynomial in $d$ for sufficiently 
large $d$ and also that homogeneous tropical ideals satisfy the ascending chain condition. Via homogenisation, one sees that both statements also hold for non-homogeneous tropical ideals (but, as in the classical setting, the theorem does not apply directly, since, for instance, when $n=1, \operatorname{in}_{(1)}\left(0 \oplus x_{1}\right)=0$ generates an ideal-the entire semiring-with a smaller Hilbert function than any tropical ideal containing $0 \oplus x_{1}$ but not 0 ).

Furthermore, Maclagan and Rincón prove that tropical ideals have tropical varieties that are finite polyhedral complexes [6, Theorem 5.11].

Theorem 2.6 If I $\subseteq R\left[x_{1}, \ldots, x_{n}\right]$ is a tropical ideal, then its (tropical) variety

$$
V(I):=\left\{\mathbf{w} \in \mathbb{R}^{n}: \mathrm{in}_{\mathbf{w}} I \text { contains no monomial }\right\}
$$

is the support of a finite polyhedral complex.

Indeed, if $I$ is homogeneous, they show that the sets of $\mathbf{w}$ where $\operatorname{in}_{\mathbf{w}} I$ is constant form the relatively open polyhedra of a polyhedral complex with support $\mathbb{R}^{n}$ called the Gröbner complex of $I$ and that the cells where $\operatorname{in}_{\mathrm{w}} I$ contains no monomial form a subcomplex with support $V(I)$. By homogeneity, all cells then contain in their lineality space the linear span of the all-ones vector 1 . In the case where $I \subseteq R\left[x_{1}, \ldots, x_{n}\right]$ is not necessarily homogeneous, let $I^{h}$ be its homogenisation in $R\left[x_{0}, x_{1}, \ldots, x_{n}\right]$. Then, $\mathbf{w} \mapsto(0, \mathbf{w})$ is a bijection between $V(I)$ and the intersection of $V\left(I^{h}\right)$ with the zeroeth coordinate hyperplane, and we give $V(I)$ the corresponding polyhedral complex structure.

The variety of a tropical ideal comes equipped with positive integral weights on its maximal polyhedra; this is inspired by [8, Lemma 3.4.7], and studied more in depth in [7].

Definition 2.7 Let $I \subseteq R\left[x_{1}, \ldots, x_{n}\right]$ be a tropical ideal, let $\sigma$ be a maximal polyhedron of $V(I)$, and let $\mathbf{w}$ be in the relative interior of $\sigma$. The multiplicity of $\sigma$ in $V(I)$ is defined as follows. First, let $I^{\prime} \subseteq R\left[x_{1}^{ \pm 1}, \ldots, x_{n}^{ \pm 1}\right]$ be the (tropical) ideal in the Laurent polynomial ring generated by $I$. After an automorphism of the Laurent polynomial ring given by $\mathbf{x}^{\mathbf{u}} \mapsto \mathbf{x}^{A \mathbf{u}}$ with $A \in \mathrm{GL}_{n}(\mathbb{Z})$, we can assume that the affine span of $\sigma$ is a translate of $\operatorname{span}\left(\mathbf{e}_{1}, \ldots, \mathbf{e}_{d}\right)$ for some $d$. In this case, by [7, Lemma 6.2], the tropical ideal $J:=\mathrm{in}_{\mathbf{w}}\left(I^{\prime}\right) \cap \mathbb{B}\left[x_{d+1}, \ldots, x_{n}\right]$ is zero-dimensional, i.e. $H_{J}(e)$ is a constant for $e \gg 0$. The multiplicity of $\sigma$ is defined to be equal to this constant, called the degree of $J$.

Remark 2.8 A more coordinate-free version of Definition 2.7 is the following. Consider the linear span of $\sigma$, defined as

$$
\operatorname{span}(\sigma):=\mathbb{R}_{\geq 0}\left\{\mathbf{v}-\mathbf{v}^{\prime} \mid \mathbf{v}, \mathbf{v}^{\prime} \in \sigma\right\} .
$$

Let $S \subseteq \mathbb{B}\left[x_{1}^{ \pm 1}, \ldots, x_{n}^{ \pm 1}\right]$ be the sub-semiring spanned by monomials $\mathbf{x}^{\mathbf{u}}$ of $\mathbf{w}$-weight $\mathbf{w} \cdot \mathbf{u}$ equal to zero for all $\mathbf{w} \in \operatorname{span}(\sigma)$. Then, $S$ itself is isomorphic to a Laurent polynomial semiring in $n-d$ variables. The multiplicity of $\sigma$ is the degree of the zero-dimensional tropical ideal $\mathrm{in}_{\mathbf{w}}\left(I^{\prime}\right) \cap S$.

We will need the following results.

Lemma 2.9 Let I be a tropical ideal in $R\left[x_{1}, \ldots, x_{n}\right]$. Denote by $I^{\prime}$ the ideal generated by $I$ in $R\left[x_{1}^{ \pm 1}, \ldots, x_{n}^{ \pm n}\right]$, and set $I^{\mathrm{sat}}:=I^{\prime} \cap R\left[x_{1}, \ldots, x_{n}\right]$. Then, $I^{\mathrm{sat}} \supseteq I$ is a tropical ideal, and $V\left(I^{\text {sat }}\right)=V(I)$ as weighted polyhedral complexes. 
We call $I^{\text {sat }}$ the saturation of $I$ with respect to $m:=x_{1} \cdots x_{n}$, and we call $I$ saturated with respect to $m$ if $I^{\text {sat }}=I$.

Proof That $I^{\text {sat }}$ is a tropical ideal containing $I$ is straightforward from the definition. Since $I^{\text {sat }} \supseteq I$, we have $V\left(I^{\text {sat }}\right) \subseteq V(I)$. Conversely, let $\mathbf{w} \in V(I)$ and $f \in I^{\text {sat }}$. Then, $\mathbf{x}^{\mathbf{u}} \odot f \in I$ for some $\mathbf{u} \in \mathbb{N}^{n}$, hence $\operatorname{in}_{\mathbf{w}}\left(\mathbf{x}^{\mathbf{u}} \odot f\right)$ is not a monomial, and therefore, neither $\operatorname{is}_{\mathbf{w}} f$. This shows that $V(I)=V\left(I^{\text {sat }}\right)$. That the multiplicities are the same follows from the fact that the multiplicities in $V(I)$ are defined using $I^{\prime}$.

If $\Sigma$ is a polyhedral complex in $\mathbb{R}^{n}$ and $\sigma$ is a polyhedron in $\Sigma$, the $\operatorname{star} \operatorname{star}_{\sigma} \Sigma$ of $\Sigma$ at $\sigma$ is a weighted polyhedral fan, whose cones are indexed by the cones $\tau$ of $\Sigma$ containing $\sigma$. The cone indexed by such $\tau$ is

$$
\bar{\tau}:=\mathbb{R}_{\geq 0}\{\mathbf{v}-\mathbf{w} \mid \mathbf{v} \in \tau \text { and } \mathbf{w} \in \sigma\},
$$

with weight equal to the weight of $\tau$ in $\Sigma$.

The following can be found in [7, Corollary 2.11 and Proposition 6.4].

Proposition 2.10 Let I be a tropical ideal in $R\left[x_{1}, \ldots, x_{n}\right]$, $\sigma$ be a polyhedron in $V(I)$, and $\mathbf{w}$ be in the relative interior of $\sigma$. Then, $\operatorname{in}_{\mathbf{w}} I \subseteq \mathbb{B}\left[x_{1}, \ldots, x_{n}\right]$ is homogeneous with respect to every vector $\mathbf{v} \in \operatorname{span}(\sigma)$ and $V\left(\operatorname{in}_{\mathbf{w}} I\right)=\operatorname{star}_{\mathbf{w}} V(I)$ as weighted polyhedral complexes.

\section{The independence complex of a tropical ideal}

Definition 3.1 Let $I \subseteq R\left[x_{1}, \ldots, x_{n}\right]$ be a tropical ideal. The independence complex of $I$ is the simplicial complex

$$
\mathcal{I}(I):=\left\{A \subseteq\{1, \ldots, n\}: I \cap R\left[x_{i}: i \in A\right]=\{\infty\}\right\}
$$

When $\mathcal{I}(I)$ is the collection of independent sets of a matroid $M$, we will say that $I$ is a matroidal tropical ideal and that $M$ is its associated algebraic matroid.

The independence complex of a tropical ideal $I$ can be recovered from its variety $V(I)$, at least if $R=\overline{\mathbb{R}}$.

Proposition 3.2 If $I \subseteq \overline{\mathbb{R}}\left[x_{1}, \ldots, x_{n}\right]$ is a tropical ideal, then

$$
\mathcal{I}(I)=\left\{A \subseteq\{1, \ldots, n\}: \pi_{A}(V(I))=\mathbb{R}^{A}\right\},
$$

where $\pi_{A}: \mathbb{R}^{n} \rightarrow \mathbb{R}^{A}$ is the coordinate projection onto the coordinates indexed by $A$. In particular, the independence complex $\mathcal{I}(I)$ depends only on the variety $V(I)$.

Proof Let $A \subseteq\{1, \ldots, n\}$. If $A \notin \mathcal{I}(I)$, then there exists $f \in I \cap \overline{\mathbb{R}}\left[x_{i}: i \in A\right]$ such that $f \neq \infty$, and $V(I) \subseteq V(f)$. We then have $\pi_{A}(V(I)) \subseteq \pi_{A}(V(f)) \subsetneq \mathbb{R}^{A}$, as claimed. For the reverse inclusion, suppose that $\pi_{A}(V(I)) \subsetneq \mathbb{R}^{A}$, and let $\mathbf{w} \in \mathbb{R}^{A} \backslash \pi_{A}(V(I))$. For any polynomial $f \in \overline{\mathbb{R}}\left[x_{1}, \ldots, x_{n}\right]$, denote by $\left.f\right|_{\mathbf{w}}$ the polynomial in $\overline{\mathbb{R}}\left[x_{i}: i \notin A\right]$ obtained by specialising each variable $x_{i}$ with $i \in A$ to $w_{i} \in \mathbb{R}$. Consider the ideal $\left.I\right|_{\mathbf{w}} \subseteq \overline{\mathbb{R}}\left[x_{i}: i \notin A\right]$ defined as $\left.I\right|_{\mathbf{w}}:=\left\{\left.f\right|_{\mathbf{w}}: f \in I\right\}$. By [7, Theorem 3.6], the ideal $\left.I\right|_{\mathbf{w}}$ is a tropical ideal. Moreover, we must have $V\left(\left.I\right|_{\mathbf{w}}\right)=\emptyset$, as any point $\mathbf{v} \in V\left(\left.I\right|_{\mathbf{w}}\right)$ would lift to the point $(\mathbf{v}, \mathbf{w}) \in V(I)$, contradicting that $\mathbf{w} \notin \pi_{A}(V(I))$. By the weak Nullstellensatz [6, Corollary 
5.17], the tropical ideal $\left.I\right|_{\mathbf{w}}$ must contain the constant polynomial 0 . But then $0=\left.f\right|_{\mathbf{w}}$ for some $f \in I$, which in particular implies that $f \in I \cap \overline{\mathbb{R}}\left[x_{i}: i \in A\right]$ and $f \neq \infty$.

Proposition 3.2 also follows from the fact that a coordinate projection of the variety of a tropical ideal is the variety of the corresponding elimination ideal [7, Theorem 4.7].

Recall that the Hilbert function $H_{I}(e)$ of a tropical ideal $I \subseteq \overline{\mathbb{R}}\left[x_{1}, \ldots, x_{n}\right]$ eventually agrees with a polynomial in $e$, called the Hilbert polynomial of $I$ [6, Proposition 3.8]. The dimension $\operatorname{dim}(I)$ of $I$ is defined as the degree of its Hilbert polynomial.

Corollary 3.3 For any tropical ideal I, we have

$$
\operatorname{dim} \mathcal{I}(I)+1=\operatorname{dim} V(I)=\operatorname{dim} I .
$$

Proof From (3.2), it is clear that $\operatorname{dim} V(I) \geq \operatorname{dim} \mathcal{I}(I)+1$. Now, if $V(I)$ contains a polyhedron $\sigma$ of dimension $d$, then there is some coordinate projection $\pi_{A}(\sigma)$ that is $d$ dimensional, and thus, from (3.1) we see that $A \in \mathcal{I}(I)$, and thus, $\operatorname{dim} \mathcal{I}(I)+1 \geq d$. This shows that $\operatorname{dim} \mathcal{I}(I)+1=\operatorname{dim} V(I)$. The equality $\operatorname{dim} V(I)=\operatorname{dim} I$ is proved in [7, Theorem 4.3].

In the classical setting, primality of an ideal implies matroidality. We do not know about a similarly appealing sufficient condition for matroidality of general tropical ideals.

Example 3.4 If $J \subseteq K\left[x_{1}, \ldots, x_{n}\right]$ is a prime ideal, where $K$ is a field with a nonArchimedean valuation, then $\operatorname{trop}(J)$ is a matroidal tropical ideal. Its associated algebraic matroid is the matroid that captures algebraic independence among the coordinate functions $x_{1}, \ldots, x_{n}$ in the field of fractions of $K\left[x_{1}, \ldots, x_{n}\right] / J$.

Problem 3.5 Find algebraic conditions on a tropical ideal that imply matroidality.

As shown in Example 3.4, any (classically) algebraic matroid is the algebraic matroid of a tropical ideal. However, in principle, it is possible that the class of matroids that are "tropically algebraic" is strictly larger than the usual class of algebraic matroids.

Question 3.6 Which matroids arise as the algebraic matroid of a tropical ideal?

\section{Quasi-products of matroids}

To motivate the definition of quasi-products, let $v_{1}, \ldots, v_{m}$ be nonzero vectors in a vector space $V$ and let $w_{1}, \ldots, w_{n}$ be nonzero vectors in a vector space $W$ over the same field. The $v_{i}$ define a matroid $M$ with ground set $[m]$ in which $S \subseteq[m]$ is dependent if and only if the set $\left\{v_{i}: i \in S\right\}$ is linearly dependent. Similarly, the $w_{j}$ define a matroid $N$ with ground set $[n]$. Now consider the vectors $v_{i} \otimes v_{j} \in V \otimes W, i \in[m], j \in[n]$. In the same manner, these define a matroid $P$ with ground set $[m] \times[n]$. One can check that $P$ is in general not determined by $M$ and $N$, i.e. the linear dependencies among the $v_{i} \otimes w_{j}$ cannot be read off from those among the $v_{i}$ and those among the $w_{j}$. However, some features of $P$ are predicted by $M$ and $N$ : for each fixed $i \in[m]$, the linear dependencies among the vectors $v_{i} \otimes w_{j}, j \in[n]$ are precisely those recorded by $N$; here we use that $v_{i}$ is nonzero. Similarly, for each $j \in[n]$, the restriction of $P$ to $[m] \times\{j\}$ is isomorphic to $M$. Furthermore, if $B$ is a basis of $M$ and $C$ is a basis of $N$, then $B \times C$ is a basis of $P$. In particular, the rank of $P$ is the product of the ranks of $M$ and $N$. Following Las Vergnas, we use these observations to define quasi-products of general matroids, as follows. 
Definition 4.1 ([5]). Let $M, N$ be loopless matroids with ground sets $[m]$, $[n]$, respectively. A quasi-product of $M$ and $N$ is a matroid $P$ with ground set $[m] \times[n]$ with the property that for each $i \in[m]$ the map $[n] \rightarrow[m] \times[n], j \mapsto(i, j)$ is an isomorphism from $M$ to the restriction of $P$ to $\{i\} \times[n]$, and for each $j \in[n]$ the map $[m] \rightarrow[m] \times[n], i \mapsto(i, j)$ is an isomorphism from $M$ to the restriction of $P$ to $[m] \times\{j\}$.

The properties of a quasi-product $P$ of $M$ and $N$ imply that if $B \subseteq[m]$ is a basis of $M$ and $C \subseteq[n]$ is a basis of $N$, then $B \times C$ is a spanning set of $P$, so the rank of $P$ is at most the product of the ranks of $M$ and $N$. By the discussion above, two matroids that are representable over the same field always admit a quasi-product whose rank is the product of their ranks. In general, however, a quasi-product with this property need not exist.

Theorem 4.2 ([5]). Any quasi-product of the rank-4 Vámos matroid $V_{8}$ and the rank-2 uniform matroid $U_{2,3}$ has rank at most $7<4 \cdot 2$.

\section{Not every Bergman fan is the variety of a tropical ideal}

We now prove that not every balanced polyhedral complex can be obtained as the variety of a tropical ideal. Our counterexample will be the Bergman fan of a matroid; see [1] for details.

Definition 5.1 Let $M$ be a loopless matroid of rank $d$ on the ground set $\{1, \ldots, n\}$. The Bergman fan $\mathcal{B}(M)$ of $M$ is the pure $d$-dimensional polyhedral fan in $\mathbb{R}^{n}$ consisting of the cones of the form

$$
\sigma_{\mathcal{F}}:=\operatorname{cone}\left(\mathbf{e}_{F_{1}}, \mathbf{e}_{F_{2}}, \ldots, \mathbf{e}_{F_{k}}\right)+\mathbb{R} \cdot \mathbf{e}_{\{1, \ldots, n\}}
$$

where $\mathcal{F}=\left\{\emptyset \subsetneq F_{1} \subsetneq F_{2} \subsetneq \ldots \subsetneq F_{k} \subsetneq\{1, \ldots, n\}\right\}$ is a chain of flats in the lattice of flats $\mathcal{L}(M)$ of $M$ and where $\mathbf{e}_{S}$ stands for the sum of the standard basis vectors $\mathbf{e}_{i}$ with $i$ running through $S$. The Bergman fan of any matroid is given the structure of a balanced polyhedral complex by defining the multiplicity of each maximal cone to be equal to 1 .

Bergman fans of matroids are the tropical linear spaces (more specifically, their part inside the torus $\mathbb{R}^{n}$ ) that correspond to valuated matroids where the basis valuations all take values in $\mathbb{B}$.

The following is our main result.

Theorem 5.2 Let $M$ be a loopless matroid of rank a with ground set $[m]$ and let $N$ be a loopless matroid of rank $b$ with ground set $[n]$. Suppose that every quasi-product of $M$ and $N$ has rank strictly less than $a \cdot b$. Then, there exists no tropical ideal $I \subseteq$ $\overline{\mathbb{R}}\left[x_{1}, \ldots, x_{m}, y_{1}, \ldots, y_{n}\right]$ such that $V(I)$ is equal to $\mathcal{B}(M \oplus N)$ as weighted polyhedral complexes, even up to common refinement.

In particular, there is no tropical ideal $I \subseteq \overline{\mathbb{R}}\left[x_{1}, \ldots, x_{3}, y_{1}, \ldots, y_{8}\right]$ such that $V(I)$ is equal to $\mathcal{B}\left(U_{2,3} \oplus V_{8}\right)$ as weighted polyhedral complexes, even up to common refinement.

Note that we do not require the polyhedral structure on $V(I)$ coming from the Gröbner complex of the homogenisation of $I$ to be equal to the fan structure on the Bergman fan described above.

To prove the theorem, in addition to the fundamental results from Sect. 2, we will need results relating $V(I)$ to $H_{I}$ for any tropical ideal $I$. 
Lemma 5.3 Let $L, L^{\prime} \subseteq R^{N}$ be tropical linear spaces. If $\operatorname{dim} L+\operatorname{dim} L^{\prime}>|N|$, then $L \cap L^{\prime} \neq\{(\infty, \ldots, \infty)\}$.

Proof The notion of stable intersection for tropical linear spaces was studied by Speyer in [12] when the underlying matroids of both tropical linear spaces were uniform matroids and later generalised by Mundinger [9] for arbitrary tropical linear spaces in $R^{N}$. The stable intersection $L \cap_{\mathrm{st}} L^{\prime}$ is a tropical linear space contained in both $L$ and $L^{\prime}$, and it has dimension a least $\operatorname{dim} L+\operatorname{dim} L^{\prime}-|N|>0$, which implies the desired result.

Proposition 5.4 Let $I \subseteq R\left[x_{1}, \ldots, x_{n}\right]$ be a tropical ideal. If the independence complex $\mathcal{I}(I)$ contains a subset $A$ of size $r$, then $H_{I}(d) \geq\left(\begin{array}{c}r+d \\ d\end{array}\right)$ for all $d \in \mathbb{N}$.

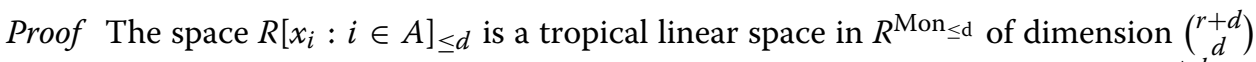
and, by assumption, it does not intersect $I_{\leq d}$. Hence, by Lemma 5.3, $\operatorname{dim} I_{\leq d} \leq\left(\begin{array}{c}n+d \\ d\end{array}\right)-$ $\left(\begin{array}{c}r+d \\ d\end{array}\right)$, and therefore, $H_{I}(d) \geq\left(\begin{array}{c}r+d \\ d\end{array}\right)$.

Proposition 5.5 Let $I \subsetneq R\left[x_{1}, \ldots, x_{n}\right]$ be a tropical ideal, and set $r:=H_{I}(1)-1$. Then, $H_{I}(d) \leq\left(\begin{array}{c}r+d \\ d\end{array}\right)$ for all $d \in \mathbb{N}$.

Proof Let $I^{h} \subseteq R\left[x_{0}, \ldots, x_{n}\right]$ be the homogenisation of $I$. Then, $\operatorname{dim}\left(I^{h}\right)_{d}=\operatorname{dim} I_{\leq d}$ for all $d \in \mathbb{N}$, and in particular $\operatorname{dim}\left(I^{h}\right)_{1}=\operatorname{dim} I_{\leq 1}=n+1-H_{I}(1)=n-r$. Moreover, by applying Theorem 2.5 with a sufficiently general weight vector $\mathbf{w}$, the Hilbert function of $I^{h}$ is also that of some monomial ideal $J$. We find that $J$ contains precisely $n-r$ of the $n+1$ variables $x_{0}, \ldots, x_{n}$, and therefore all their multiples. This implies that $\operatorname{dim} J_{d} \geq\left(\begin{array}{c}n+d \\ d\end{array}\right)-\left(\begin{array}{c}r+d \\ d\end{array}\right)$, where the last term counts monomials in the remaining $r+1$ variables of degree $d$. We then have

$$
H_{I}(d)=\left(\begin{array}{c}
n+d \\
d
\end{array}\right)-\operatorname{dim} I_{\leq d}=\left(\begin{array}{c}
n+d \\
d
\end{array}\right)-\operatorname{dim} J_{d} \leq\left(\begin{array}{c}
n+d \\
d
\end{array}\right)-\left(\begin{array}{c}
n+d \\
d
\end{array}\right)+\left(\begin{array}{c}
r+d \\
d
\end{array}\right),
$$

as desired.

The following proposition shows that the algebraic matroid of a Bergman fan $\mathcal{B}(M)$ (as in Proposition 3.2) is equal to the matroid $M$.

Proposition 5.6 ([14, Lemma 3]). The independence complex of the Bergman fan $\mathcal{B}(M)$ of a loopless matroid $M$ is the same as the independence complex of $M$.

We now present a key step towards proving our main result.

Proposition 5.7 Let $M$ be a loopless matroid on the ground set $\{1, \ldots, n\}$. Suppose $J \subseteq \mathbb{B}\left[x_{1}, \ldots, x_{n}\right]$ is a homogeneous tropical ideal, saturated with respect to $x_{1} \cdots x_{n}$, whose variety $V(J)$ has a common refinement, as weighted polyhedral complexes, with the Bergman fan $\mathcal{B}(M)$ (with weight 1 in all its maximal cones). Then, the matroid $M\left(J_{1}\right)$ is equal to $M$, under the identification $x_{i} \leftrightarrow$ i of ground sets.

Proof Let $B=\left\{b_{1}, \ldots, b_{d}\right\}$ be a basis of $M$. For $0 \leq i \leq d$, consider the flat $F_{i}$ of $M$ obtained as the closure of the set $\left\{b_{1}, \ldots, b_{i}\right\}$, and let $\sigma$ be the maximal cone of $\mathcal{B}(M)$ corresponding to the chain of flats $\emptyset=F_{0} \subsetneq F_{1} \subsetneq \cdots \subsetneq F_{d-1} \subsetneq F_{d}=\{1, \ldots, n\}$. Let $\tau \subseteq \sigma$ be a maximal cone in a common refinement of both $V(J)$ and $\mathcal{B}(M)$. The linear $\operatorname{span} \operatorname{span}(\tau)=\operatorname{span}(\sigma)$ consists of all vectors $\mathbf{w} \in \mathbb{R}^{n}$ for which $w_{i}=w_{j}$ whenever $\{i, j\} \subseteq$ 
$F_{k} \backslash F_{k-1}$ for some $k=1, \ldots, d$. A monomial $\mathbf{x}^{\mathbf{u}}$ in $\mathbb{B}\left[x_{1}^{ \pm 1}, \ldots, x_{n}^{ \pm 1}\right]$ has $\mathbf{w}$-weight equal to zero for all such $\mathbf{w}$ if and only if for every $k$ we have $\sum_{i \in F_{k} \backslash F_{k-1}} u_{i}=0$. As in Remark 2.8, let $S$ be the subsemiring of $\mathbb{B}\left[x_{1}^{ \pm 1}, \ldots, x_{n}^{ \pm 1}\right]$ consisting of all polynomials involving only such monomials, and let $J^{\prime}$ be the (tropical) ideal in $\mathbb{B}\left[x_{1}^{ \pm 1}, \ldots, x_{n}^{ \pm 1}\right]$ generated by $J$.

Take $\mathbf{v}$ to be a vector in the relative interior of $\tau$. Since $\tau$ has multiplicity 1 in $V(J)$, $\operatorname{in}_{\mathbf{v}}\left(J^{\prime}\right) \cap S$ is zero-dimensional of degree 1 and contains no monomials. Hence, for any pair of distinct monomials $\mathbf{x}^{\mathbf{u}}, \mathbf{x}^{\mathbf{u}^{\prime}}$ in $S, \operatorname{in}_{\mathbf{v}}\left(J^{\prime}\right) \cap S$ contains the binomial $\mathbf{x}^{\mathbf{u}} \oplus \mathbf{x}^{\mathbf{u}^{\prime}}$. In particular, if $\{i \neq j\} \subseteq F_{k} \backslash F_{k-1}$ for some $k$ then $0 \oplus x_{i}^{-1} x_{j} \in \operatorname{in}_{\mathbf{v}}\left(J^{\prime}\right) \cap S$, and thus, $x_{i} \oplus x_{j} \in \operatorname{in}_{\mathbf{v}}\left(J^{\prime}\right)$. As $J$ is homogeneous and saturated with respect to $x_{1} \cdots x_{n}$, this implies that there is a polynomial of the form $x_{i} \oplus x_{j} \oplus f$ in $J_{1}$ where $f$ is a sum of variables all contained in $F_{k-1}$. It follows that $x_{i}$ is in the closure of $F_{k-1} \cup\left\{x_{j}\right\}$ in the matroid $M\left(J_{1}\right)$. We conclude that $\left\{b_{1}, \ldots, b_{d}\right\}$ is a generating set in the matroid $M\left(J_{1}\right)$, and thus, $\operatorname{rank}\left(M\left(J_{1}\right)\right) \leq \operatorname{rank}(M)$. Now, the tropical prevariety cut out by the linear polynomials in $J$ is equal to $\mathcal{B}\left(M\left(J_{1}\right)\right)$, so we have $\mathcal{B}\left(M\left(J_{1}\right)\right) \supseteq V(J)=\mathcal{B}(M)$. It follows from [11, Lemma 7.4] that $\mathcal{B}\left(M\left(J_{1}\right)\right)=\mathcal{B}(M)$, and thus, $M\left(J_{1}\right)=M$, completing the proof.

We conclude with the proof of the main theorem.

Proof of Theorem 5.2 Suppose that such an $I$ exists, and denote $O:=M \oplus N$. We first argue that we may replace $I$ by an ideal $J$ that is homogeneous as well as saturated. To this end, let $\sigma$ be a polyhedron in $V(I)$ whose affine span is $\mathbb{R} \cdot \mathbf{1}$ (which is contained in the lineality space of $\mathcal{B}(O)$ ), and let $\mathbf{w}$ be in the relative interior of $\sigma$. Set $J^{\prime}:=\operatorname{in}_{\mathbf{w}} I \subseteq \mathbb{B}\left[x_{1}, \ldots, x_{m}, y_{1}, \ldots, y_{n}\right]$. By Proposition 2.10, the tropical ideal $J^{\prime}$ is homogeneous (with respect to 1 ) and has variety $V\left(J^{\prime}\right)=\operatorname{star}_{\mathbf{w}} V(I)$, which is equal to $\mathcal{B}(O)$ up to common refinement. Consider the homogeneous ideal $J:=\left(J^{\prime}\right)^{\text {sat }}$. By Lemma 2.9, we have that $V(J)$ is also equal to $\mathcal{B}(O)$ up to common refinement.

Now, by Proposition 5.7, $M\left(J_{1}\right)$ is equal to $O$. Since rk $O=a+b$, we find that $H_{J}(1)=$ $1+a+b$, and thus, by Proposition 5.5, $H_{J}(d) \leq\left(\begin{array}{c}a+b+d \\ d\end{array}\right)$ for all $d$. On the other hand, since $V(J)=\mathcal{B}(O)$, by Propositions 5.6 and 3.2 the tropical ideal $J$ is matroidal, with associated algebraic matroid $O=M \oplus N$. Hence, by Proposition 5.4 we have $H_{J}(d) \geq\left(\begin{array}{c}a+b+d \\ d\end{array}\right)$. We conclude that $H_{J}(d)=\left(\begin{array}{c}a+b+d \\ d\end{array}\right)$.

Denote $Q:=M\left(J_{2}\right)$. The matroid $Q$ has rank $H_{J}(2)-H_{J}(1)=\left(\begin{array}{c}a+b+1 \\ 2\end{array}\right)$ on the ground set $S_{1} \sqcup S_{2} \sqcup S_{3}$, where $S_{1}:=\left\{x_{i} x_{j} \mid 1 \leq i \leq j \leq m\right\}, S_{2}:=\left\{y_{i} y_{j} \mid 1 \leq i \leq j \leq n\right\}$, and $S_{3}:=\left\{x_{i} y_{j} \mid 1 \leq i \leq m, 1 \leq j \leq n\right\}$. The restriction $Q \mid S_{1}$ is spanned by all products of two elements in a basis of $M\left(J_{1}\right) \mid\left\{x_{1}, x_{2}, \ldots, x_{m}\right\}$, and hence has rank at most $\left(\begin{array}{c}a+1 \\ 2\end{array}\right)$. Similarly, the restriction $Q \mid S_{2}$ has rank at most $\left(\begin{array}{c}b+1 \\ 2\end{array}\right)$. Hence, $Q \mid S_{3}$ has rank at least $\left(\begin{array}{c}a+b+1 \\ 2\end{array}\right)-\left(\begin{array}{c}a+1 \\ 2\end{array}\right)-\left(\begin{array}{c}b+1 \\ 2\end{array}\right)=a b$.

Since $J$ is saturated, for each $1 \leq i \leq m$, multiplication by $x_{i}$ yields an isomorphism between the matroid $M\left(J_{1}\right) \mid\left\{y_{1}, \ldots, y_{n}\right\} \cong N$ and the restriction of $Q$ to $x_{i} \cdot\left\{y_{1}, \ldots, y_{n}\right\} \subseteq$ $S_{3}$. Similarly, for each $1 \leq j \leq n$, the restriction of $Q$ to $y_{j} \cdot\left\{x_{1}, \ldots, x_{m}\right\}$ is isomorphic to $M$. Hence, $Q \mid S_{3}$ is a quasi-product of $M$ and $N$ in the sense of Definition 4.1. But the assumption in the theorem is that such a quasi-product has rank strictly less than $a \cdot b$, a contradiction. Hence, no such ideal $I$ exists.

The second part of the main theorem is a direct consequence of the first part and Theorem 4.2 by Las Vergnas. 


\section{Concluding remarks}

Using the result by Las Vergnas that $U_{2,3}$ and $V_{8}$ do not have a quasi-product of rank 8 , we have showed that the Bergman fan of their direct sum is not the tropical variety of any tropical ideal, with weight 1 on all the maximal cones.

We do not know whether there exists a tropical ideal whose tropical variety is the Bergman fan of $U_{2,3} \oplus V_{8}$ as a set, without the condition that all weights be 1 .

We also do not know whether $\mathcal{B}\left(V_{8}\right)$ itself is the tropical variety of any tropical ideal with weight one on the maximal cones. To study this question for a matroid $M$, one needs to develop the theory of symmetric squares of matroids, in a fashion similar to Las Vergnas's quasi-products from Sect. 4. But already for $V_{8}$, this seems considerably harder than quasi-products of $U_{2,3}$ with $V_{8}$.

Finally, we'd like to point out that for any $m \geq 3$, the matroids $U_{2, m}$ and $V_{8}$ do not admit a quasi-product of rank 8 . Indeed, if $P$ were such a quasi-product on $[m] \times[8]$, then for any basis $C \subseteq[8]$ of $V_{8}$ the set [2] $\times C$, which spans $P$, would have to be a basis. But then the restriction of $P$ to [3] $\times$ [8] would be a quasi-product of $U_{2,3}$ and $V_{8}$ of rank 8 , a contradiction to Las Vergnas's Theorem 4.2. This simple observation yields infinitely many matroids to which our Theorem 5.2 applies. However, it would be interesting to find more intricate families of pairs of matroids that do not admit quasi-products of the correct rank.

\section{Acknowledgements}

Both authors would like to thank the Mittag-Leffler Institute for their hospitality during the Spring 2018 program, when this paper was conceived. In addition, FR would like to thank the Discrete Mathematics/Geometry Group at TU Berlin for their support while this paper was written.

Funding

Open Access funding provided by Universität Bern.

\section{Author details}

${ }^{1}$ Universität Bern, Mathematisches Institut, Sidlerstrasse 5, 3012 Bern, Switzerland, '2Eindhoven University of Technology, Eindhoven, Netherlands, ${ }^{3}$ School of Mathematical Sciences, Queen Mary University of London, Mile End Road, London E1 4NS, United Kingdom.

Received: 30 November 2020 Accepted: 17 May 2021 Published online: 28 June 2021

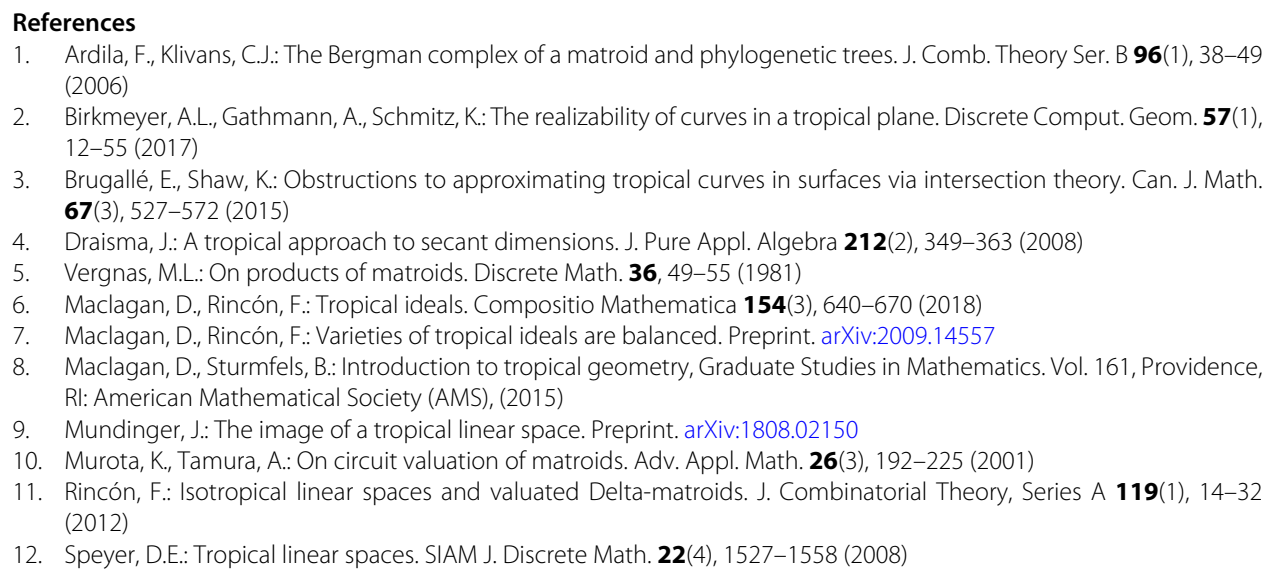


13. Speyer, D.E.: Parameterizing tropical curves I: Curves of genus zero and one. Algebra Number Theory 8(4), 963-998 (2014)

14. Josephine, Yu.: Algebraic matroids and set-theoretic realizability of tropical varieties. J. Comb. Theory Series A 147 41-45 (2017)

\section{Publisher's Note}

Springer Nature remains neutral with regard to jurisdictional claims in published maps and institutional affiliations. 